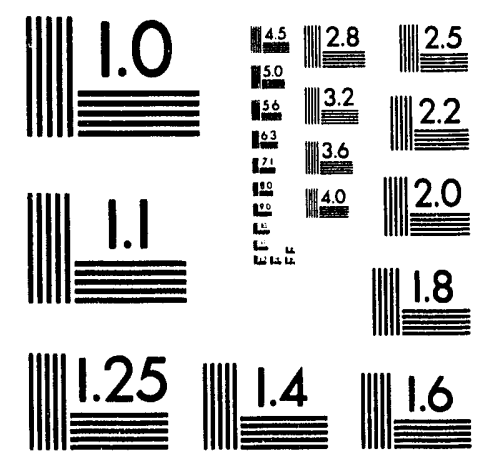



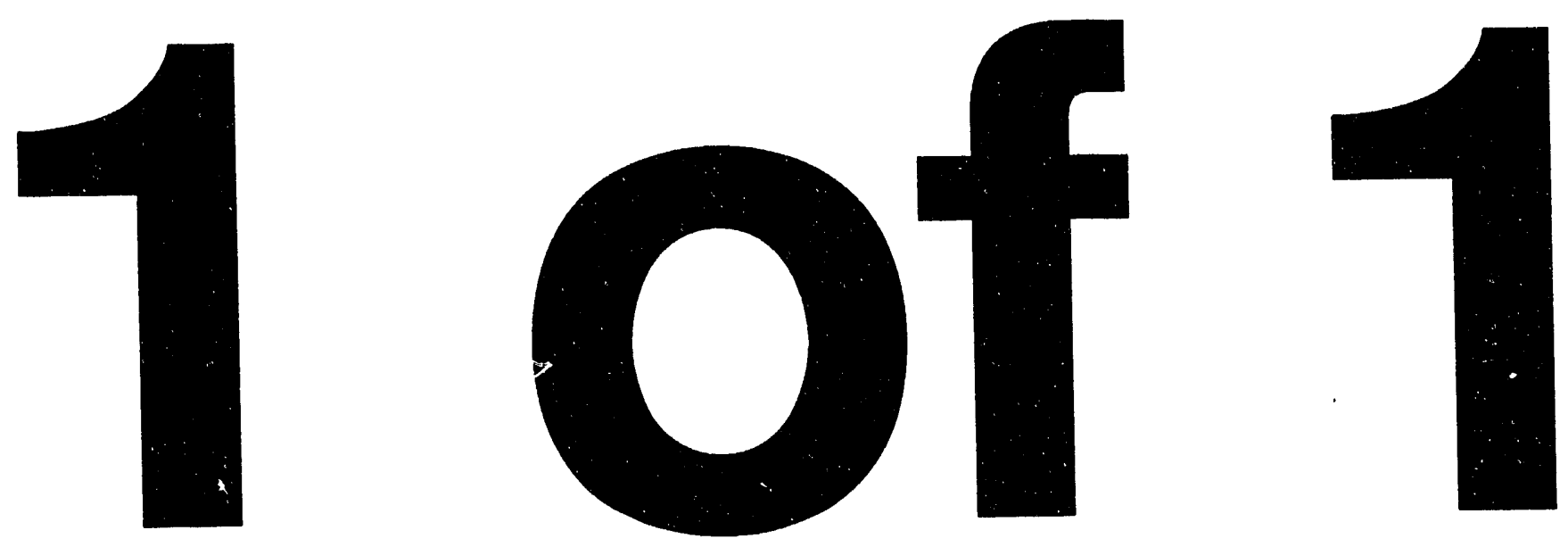


\section{RESONANCE RAMAN AND PHOTOPHYSICAL STUDIES OF TRANSITION METAL COMPLEXES IN SOLUTION AND ENTRAPPED IN ZEOLITES}

Progress Report

for Period Aug. 1, 1990 - January, 1993

James R. Kincaid

Chemistry Department

Marquette University

Milwaukee, WI 53233

Prepared for the

U.S. Department of Energy

Office of Basic Energy Sciences

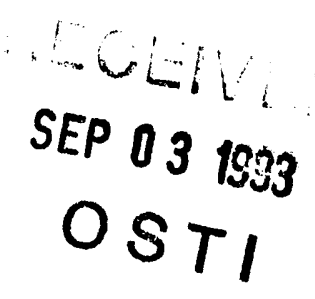

\section{NOT I C E}

This report was prepared as an account of work sponsored by the United States Government. Neither the United States nor the Department of Energy, nor any of their employees, nor any of their contractors, subcontractors, or their employees, makes any warranty, express or implied, or assumes any legal liability or responsibility for the accuracy, completeness, or usefulness of any information, apparatus, product or process disclosed or represents that its use would not infringe privately-owned rights.

\section{MASTER}




\section{A. ABSTRACT}

This report provides a concise summary of the work conducted during the first two years and four months of the current three year grant. In the proposal which described the three year plan, three major areas of interest were identified. The area of most intense activity involves the study of the effects of entrapment of ruthenium(II)-polypyridyl complexes within the supercages of Y-zeolite. The manuscripts and on-going projects which deal with this issue are described in part B.1 of this report, and, as was anticipated and discussed in the original application, the results of the initial series of studies document alterations in the inherent photophysical properties of the entrapped complexes and provide insight into the factors responsible for these changes.

A second major activity proposed in the original document was to undertake an investigation of the feasibility of entrapment of metallophthalocyanines within the Y-zeolite supercages. As is described in part B.2 of this report, initial problems in the preparative methodology used for these systems have been overcome and several systems have now been prepared and purified so as to be useful for projected photoredox studies.

Finally, a third major objective of the proposal was to develop and utilize a sound interpretive framework for resonance Raman (RR) and time-resolved RR (ie., $T R^{3}$ ) methods for investigating the subtle perturbations in the ground-and ${ }^{3} \mathrm{MLCT}$-state electronic structure which might be induced by substituent or environmentally-induced asymmetry, effects which could possibly lead to critical functional alterations. The several manuscripts resulting from this phase of the work are summarized in section B. 3 of this report. 


\section{B. Summary of Results}

\section{Zeolite-Entrapped Ruthenium(In) Complexes of Bipyridine and Related Ligands}

a. "Synthesis and Spectroscopic Properties of Zeolite-Entrapped Bis-Heteroleptic Ruthenium(II) Polypyridine Complexes"; K. Maruszewski, D.P. Strommen, K. Handrich and J.R. Kincaid; Inorganic Chemistry, 30, 4579 (1991).

A convenient method for the preparation of polypyridine complexes of $\mathrm{Ru}(\mathrm{II})$ within the supercages of zeolite- $Y$ was developed. The method is general in the sense that it permits a large variety of heteroleptic complexes to be prepared via formation and isolation of bis-homoleptic precursors (ie., Z- $\mathrm{RuL}_{2}{ }^{2+}$ ) to which a third (different) ligand can be added to form $\mathrm{Z}-\mathrm{RuL}_{2} \mathrm{~L}^{2+}$. As part of this initial work several bis-heteroleptic, tris-ligated complexes were prepared and effecient analytical procedures were developed to confirm purity. Using the methodology developed in this work, two series of complexes were prepared and studied as described below.

b. $\quad$ "Zeolite-Entrapped Ruthenium(II) Complexes with Bipyridine and Related Ligands. Elimination of Ligand-Field State Deactivation and Increases in ${ }^{3}$ MLCT-State Lifetimes"; K. Maruszewski, D.P. Strommen and J.R. Kincaid. J. Am. Chem. Soc., manuscript in revision.

The most important goal of the original proposal was to provide a thorough documentation and explanation of the effects of the zeolite supercage on the photophysical and photochemical properties of a variety of judiciously chosen polypyridine complexes. The above referenced manuscript represents such a study of the first two series of target complexes. 
Series 1

$\mathrm{Z}-\mathrm{Ru}(\mathrm{bpy})_{3}{ }^{2+}$

Z-Ru(bpy) ${ }_{2}(\mathrm{dmb})^{2+}$

$\mathrm{Z}-\mathrm{Ru}(4-\mathrm{mmb})_{3}{ }^{2+}$

where

$\mathrm{dmb}=4,4$ '-dimethyl-2,2'-bpy

$4-\mathrm{mmb}=4-\mathrm{methyl}-2,2$ '-bpy
Series 2

$\mathrm{Ru}(\mathrm{bpz})_{3}{ }^{2+}$

$\mathrm{Ru}(\mathrm{bpy})_{2}(\mathrm{bpz})^{2+}$

where

$\mathrm{bpz}=2,2$ '-bipyrazine

In the first series, the steric bulk of the entrapped complex is systematically varied whereas the second series includes ligands which bear peripheral nitrogen atoms which may interact (acid/base interactions) with the zeolite framework. The essential results are concisely summarized as follows. Careful measurements of the temperature-dependent lifetimes revealed that for all of the complexes studied the so-called dd-state (thermally activated) decay pathway is essentially eliminated. This is entirely consistent with a similar deactivation observed by Meyer and coworkers for $\mathrm{Ru}(\mathrm{bpy})_{3}{ }^{2+}$ embedded in a cellulose acetate matrix. In the present case, supercage constriction of the dd-state $\mathrm{Ru}-\mathrm{N}$ bond length raises the energy of this state to a point where thermal population becomes insignificant and the decay through the so-called "4th MLCT state" dominates the thermally activated processes. This, of course, has important implications for catalyst design in the sense that photochemical ligand loss (ie., eventual loss of sensitizer) is eliminated by encapsulation within the zeolite framework.

While no evidence is obtained for alteration of ligand orbitals for the first series of complexes, the two bipyrazine containing complexes exhibit significanct blue shifts in their emission maxima upon entrapment within the zeolite cages, In addition, for both complexes, 
the lifetimes at room temperature show significant increases (by factors of approximately 3 and 2.).

Clearly, these results represent the achievement of one of the three essential goals of the proposal and justify continuation of further work in this area, the details of which are provided in the accompanying proposal.

\section{Zeolite-Entrapped Metallophthalocyanines}

a. Synthesis

A good deal of time in the initial stages of this contract period was spent in familiarizing ourselves with methods to produce and purify zeolite particles which contain various metallophthalocyanines (MPc) entrapped within the supercages. The major problem encountered was the apparent inefficiency of methods to remove surface coated MPc's by soxhlet extraction. Actually, much effort was wasted owing to the failure of one of the main reference works to point out the superiority of chloro-naphthalene as the most effective solvent. Eventually, the procedure outlined below was developed.

$$
\begin{aligned}
& Z+M^{n^{+}} \longrightarrow Z-M^{n^{+}} \stackrel{250^{\circ} \mathrm{C}}{\longrightarrow}>\operatorname{dry} Z-M^{n^{+}} \frac{X S D C B *}{8 \text { fold }}>\text { Crude Z-MPc } \\
& \mathrm{Z}-\mathrm{MPc} \text { (crude) } \stackrel{\text { Acetone }}{\longrightarrow} \stackrel{\text { Pyridine }}{\longrightarrow} \stackrel{\mathrm{Cl}-\mathrm{Nap}}{\longrightarrow} \stackrel{\mathrm{EtOH}}{\longrightarrow}>\mathrm{Z}-\mathrm{MPc} \\
& \text { * DCB is Dicyanobenzene }
\end{aligned}
$$

Alternatively, in some cases (eg Z-NiPc), the following sublimation is effective, using a quartz tube and furnace

$$
Z-M P c \text { (Crude) } \underset{\text { Flowing } \mathrm{He}}{\stackrel{400^{\circ} \mathrm{C}}{\longrightarrow}} \mathrm{Z}-M P c+M P c
$$


where the MPc solid condenses outside of the tube furnace on the walls of the tube.

b. Characterization

Various spectroscopic methods were used to characterize the purified Z-MPc particles. The reflectance spectra of the various samples (and reference compounds) were obtained. In Figure 2.1, it can be secn that the "crude" (ie., surface coated material) exhibits a reflectance spectrum which is quite similar to that of powdered MPc. However, the washed (purified) sample exhibits a unique spectrum. While the broadened spectral features of the zeolite entrapped material might be ascribed to structure perturbations of the macrocycle induced by the constraints of the supercage, the observed RR spectra show no substantial alterations (Figure 2.2).

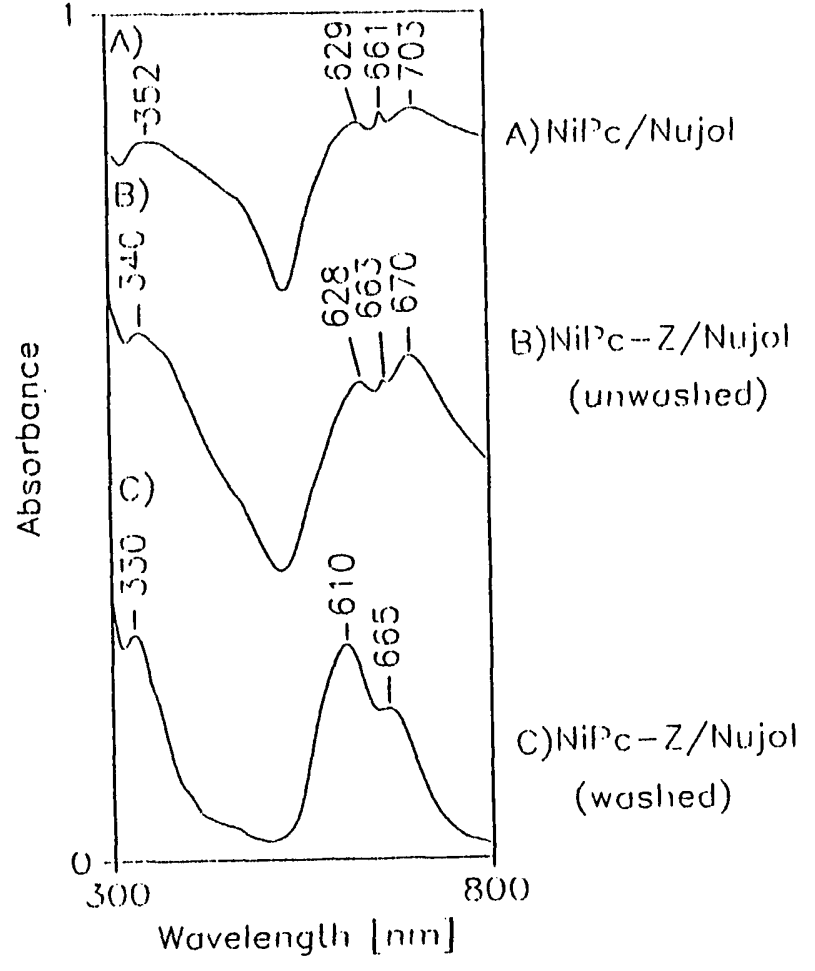

Fig. 2.1

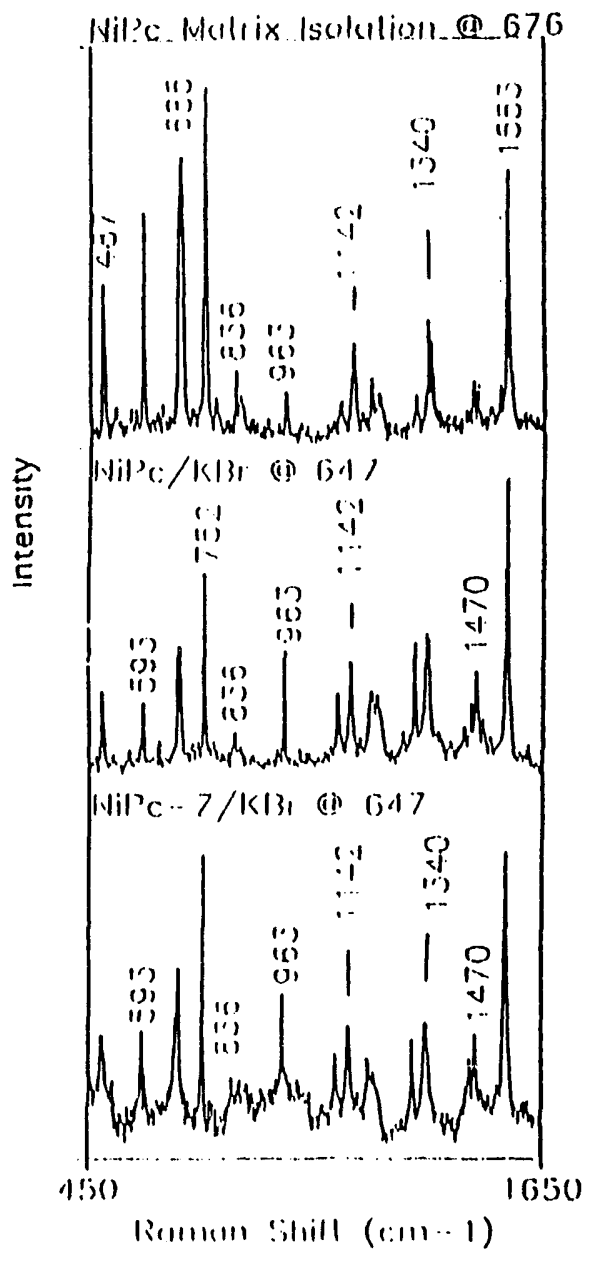

Fig. 2.2 
The above results, provide convincing evidence that various MPc can be synthesized and trapped within the $13 \AA$ supercage and that there are no apparent gross structural perturbations, an indication that the inherent photophysical properties may not be substantially altered. These materials are therefore suitable for the various photoredox studies described in the accompanying proposal.

Inasmuch as the primary photoredox product following oxidative quenching by acceptors in an adjacent cage is the $\mathrm{MPc}^{+}$cation-radical, it is important to document the RR spectrum of this species. As is shown in Figure 2.3, the RR spectrum of the MPc ${ }^{+}$is distinctly different from that of the neutral parent MPc, a fact which will enable us to document oxidative quenching processes during the photoredox studies of the acceptorloaded Z-MPc particles as described in the accompanying proposal.

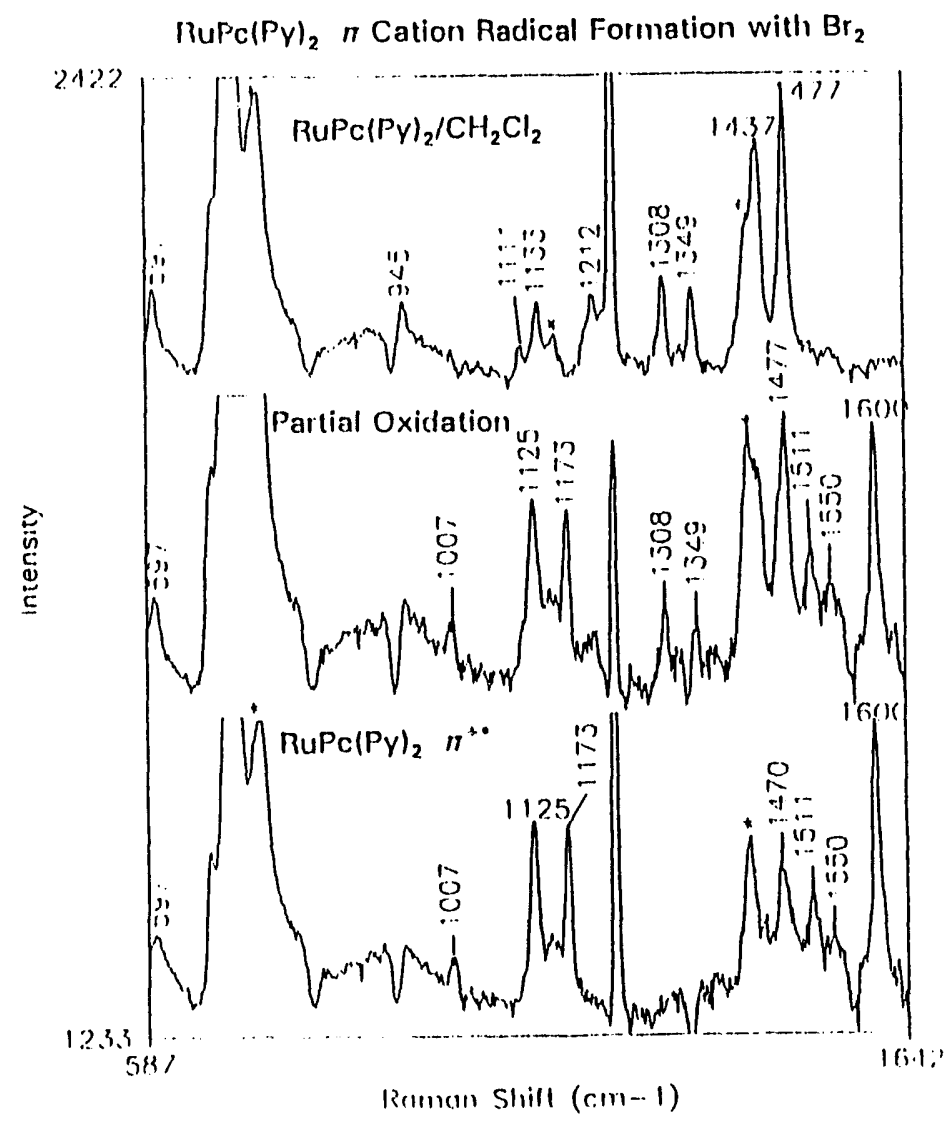

Fig. 2.3 
3. Resonance Raman (RR) and Time-Resolved Resonance Raman (TR ${ }^{3}$ ) Studies of the Nature of ${ }^{3}$ MLCT States of Ru(II)-Polypyridine Complexes

a. $\quad$ "Resonance Raman Spectra of Ru(bipyrazine ${ }_{3}{ }^{2+}$ in Aqueous Sulfuric Acid Solutions"; G.D. Danzer and J.R. Kincaid; J. Raman. Spectroscopy 23 681-690 (1992)

The RR spectra of $\mathrm{Ru}(\mathrm{bpz})_{3}{ }^{2+}$ were obtained in solutions of $\mathrm{H}_{2} \mathrm{SO}_{4} / \mathrm{H}_{2} \mathrm{O}$ and ${ }^{2} \mathrm{H}_{2} \mathrm{SO}_{4} /{ }^{2} \mathrm{H}_{2} \mathrm{O}$ at acid concentrations ranging between 0 and $98 \%$ (by weight). The results provided the first evidence for vibrational isolation of individual fragments of these chelate ligands and the interpretation of the RR spectra at various acid concentrations was consistent with sequential protonation (or deuteriation) of the six peripheral nitrogen atoms, the first three protons (deuterons) being added to separate bipyrazine ligands. These conclusive findings are in agreement with previous suggestions based on the results of absorption and emission spectral studies.

b. $\quad$ Resonance Raman and Time-Resolved Resonance Raman Evidence for EnhancedLocalization in the ${ }^{3}$ MLCT States of Ruthenium(II) Complexes with the Inherently Asymmetric Ligand, 2,2'-Pyridylpyrazine." G. Danzer, J. Golus and J.R. Kincaid; J. Am. Chem. Soc., submitted.

$R R$ and $T R^{3}$ spectra of $R u(p y p z)_{3}{ }^{2+}$ and its selectively deuteriated analogue $R u(3,5-$ d2pypz $)_{3}{ }^{2+}$ were obtained. Comparison of the spectral data with those of judiciously chosen reference compounds confirms the fact that the spectrum of the ground state species is interpretable in terms of vibrationally isolated fragments with the exception of several modes which involve the inter-ring stretch and adjacent bonds. More importantly, the $\mathrm{TR}^{3}$ spectra of the ${ }^{3}$ MLCT state are shown to be consistent with the presence of a coordinated (pypz) in which the electronic charge is highly polarized towards the pyrazine fragment of the chelate. 
This study not only illustrates the impressive utility of $\mathrm{RR}$ and $\mathrm{TR}^{3}$ for detailed interrogation of ${ }^{3}$ MLCT states of such species but also clearly demonstrates that extra-localization effects are important in dictating the precise nature of such states. This is obviously an important issue for the design and elucidation of the nature of organized assemblies which incorporate asymmetric ligands (or which give rise to an environmentally-induced asymmetry).

c. "Resonance Raman and Time-Resolved Resonance Raman Studies of Ruthenium(II) Complexes with 4-Methyl-2,2'-Bipyridine. Evidence for Asymmetric Charge Distribution in the ${ }^{3}$ MLCT Excited State"; S.M. Treffert, J. Golus, D.P. Strommen and J.R. Kincaid; J. Phys. Chem., submitted.

This study was undertaken in order to determine if a relatively small perturbation in the $\pi$-orbital energies of the two fragments of the chelate could give rise to enhanced localization effects; ie., the relative energies of the orbitals are closer for 4-methyl-2,2'bipyridine than in the case of pyridylpyrazine. Again, careful RR and $T^{3}$ measurements were made for the parent complex, $\mathrm{Ru}(\mathrm{mmb})_{3}{ }^{2+}$, as well as for several selectively deuteriated analogues and appropriate reference complexes. In this case too, definitive evidence is obtained from the $\mathrm{TR}^{3}$ spectra for polarization of electron density towards the pyridine fragment of the 4-mmb "radical-like" ligands.

\section{Peripheral Studies}

Grouped in this section are brief summaries of studies of several issues which, though not specifically targeted in the orginal application, were pursued as opportunities arose because of their percieved fundamental importance.

a. "Position-Dependant Deuteriation Effects on the Non-Radiative Decay of the ${ }^{3}$ MLCT State of tris -Bipyridine Ruthenium (II) and the Identification of the Critical Acceptor Mode" K. Maruszewski, K. Bajdor, K.P. Strommen, and J.R. Kincaid; in revision, to be resubmitted to J. Phys. Chem. 
The availability of a large number of selectively deuteriated analogues of $R u(b p y)_{3}{ }^{2+}$, coupled with the previous observation of deuteriation effects on non-radiative decay rate constants, prompted us to undertake a systematic investigation of this issue. The extensive data on the lifetimes for all of the deuteriated analogues were interpreted with the aid of a detailed vibrational analysis and a well-established theoretical framework previously applied to the parent complex by Meyer, Casper, and coworkers. Careful analysis of the results documents position-dependent deuteriation effects and reveals that there is only one totally symmetric vibration $\left(\nu_{8}\right)$ whose frequency shifts upon deuteriation correlate with the observed variations in lifetimes. This work thus represents an experimental verification of the theorybased prediction of a single, dominant vibrational decay pathway and provides convincing evidence for identification of the "critical acceptor mode" as the $\nu_{8}$ mode which occurs near $1450 \mathrm{~cm}^{-1}$.

\section{b. Inverse Deuteriation Effects on ${ }^{3}$ MLCT State Lifetimes}

As part of a $\mathrm{TR}^{3}$ study of heteroleptic complexes (vide infra) it was necessary to prepare $4,4^{\prime}, 5,5^{\prime}-\mathrm{C}^{2} \mathrm{H}_{3}$-bipyridine $\left(\mathrm{d}_{12} \mathrm{TMB}\right)$. It was found that the lifetime of this deuteriated $\mathrm{Ru}(\mathrm{II})$ analogue was actually significantly shorter than that of the natural abundance species. Thus, $\tau\left[\mathrm{RuTMB}_{3}{ }^{2+}\right]=560$ ns while $\tau\left[\mathrm{Ru}\left(\mathrm{d}_{12} \mathrm{TMB}\right)_{3}{ }^{2+}\right]=470 \mathrm{~ns}$. Interestingly, the RR spectra of the two complexes document a shift to higher frequency for one of the RR active modes in the deuteriated analogue relative to its value in the spectrum of $\mathrm{Ru}(\mathrm{TMB})_{3}{ }^{2+}$. As is discussed in the accompanying proposal, this issue will be pursued to determine if further evidence for the existence of a dominant acceptor mode can be documented. 
c. "Resonance Raman and Time-Resolved Resonance Raman Studies of ${ }^{3}$ MLCT-State Population in the Series of Ruthenium (II) Complexes with Bipyridine and tetraMethyl Bipyridine" G.D. Danzer, K. Bajdor, and J.R. Kincaid, J. Raman Spectroscopy, in press.

The RR and $T R^{3}$ spectra of the complexes of Ru(II) with bpy and TMB were obtained. The RR spectra of the ground state species show no anomalous behavior and are consistent with vibrationally isolated chelate ligands. $T$ he $\mathrm{TR}^{3}$ results are consistent with specific population of a bpy localized ${ }^{3}$ MLCT state in both of the heteroleptic complexes, $\mathrm{Ru}(\mathrm{bpy})(\mathrm{tmb})_{2}{ }^{2+}$ and $\mathrm{Ru}(\mathrm{bpy})_{2}(\mathrm{tmb})^{2+}$. Importantly, even in the case of $\mathrm{Ru}(\mathrm{bpy})(\mathrm{tmb})_{2}{ }^{2+}$ there is no evidence for formation of $a^{3} \mathrm{MLCT}$ state formulated as $\left[\mathrm{Ru}^{3+}(\mathrm{bpy})(\mathrm{tmb})\left(\mathrm{tmb} \mathrm{b}^{-}\right)\right]^{2+}$. This is in contrast to the corresponding case for the dimethylbipyridine/bpy complex,

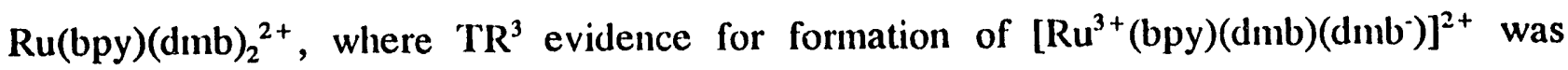
obtained, though it comprised only a small fraction of the total ${ }^{3}$ MLCT-state population. Apparently, while dimethyl substitution is insufficient to prevent population of the alkylated ligand, tetramethylation appears to lead to quite specific population of the bpy-centered ${ }^{3}$ MLCT state. These results are of interest inasmuch as alkylated-bpy's are being increasingly used to construct supramolecular assemblies wherein the detailed interpretation of the kinetics can be greatly facilitated by a knowledge of the relative populations of given states.

\section{Comment on Personnel}

It seems appropriate to provide information regarding a personnel crisis which arose during the course of this contract. In the second year, two key senior coworkers left the group, Dr. Dennis P. Strommen who moved to Idaho State University and Dr. Gerald Danzer who no longer participates full-time, but only one day/week (full-time summers). 
Also, a third member, Dr. K. Maruszweski had to leave temporarily (4 months). This left the group with only two, relatively inexperienced graduate students and resulted in a lag in productivity and delayed completion of several projects which were nearly completed. The situation has now been rectified as evidenced by the recent completion and submission of five manuscripts, with several more projected before the expiration of the current contract.

\section{Publications which cite ER 13619}

1. K. Maruszev . xi, D.P. Strommen, K. Handrich and J.R. Kincaid. "Synthesis and Spetroscopic Properties of Zeolite-Entrapped Bis-Heteroleptic Ruthenium(II) Polypyridine Complexes", Imorganic Chemistry, 30, 4579 (1991).

2. G.D. Danzer and J.R. Kincaid. "Resonance Raman Studies of Ru(bipyrazine) ${ }_{3}{ }^{2+}$ in Aqueous Sulfuric Acid Solution", J. Raman Spectrosc., 23, 681, (1992).

3. C.S. Johnson, C. Mottley, J.T. Hupp and G.D. Danzer. "Resonance Raman Spectroscopic Studies of Trans-Dioxorhenium(V) tetrapyridyl Species" Inorganic Chemistry, $\underline{31}, 5143,1992$.

4. G.D. Danzer, J.A. Golus and J.R. Kincaid. "Resonance Raman and Time-Resolved Resonance Raman Evidence for Enhanced-Localization Effects in Ruthenium(II) Complexes of Inherently Asymmetric Ligand, 2,2'-Pyridylpyrazine", J. Am Chem. Soc., submitted.

5. K. Maruszewski, D.P. Strommen and J.R. Kincaid. "The Structure and Photophysics of Zeolite-Entrapped Complexes of Ruthenium(II) with Bipyridine and Related Ligands" J. Am Chem. Soc., submitted.

6. S. Treffert, G.D. Danzer, D.P. Strommen and J.R. Kincaid. "The ${ }^{3}$ MLCT Excited State Structure of Ruthenium Complexes of 4-methyl-2,2'-bipyridine. Enhanced Localization of Excited State Electron Density on the Pyridine-Fragment", J. Phys. Chem., submitted.

7. K. Maruszewski, D.P. Strommen and J.R. Kincaid. "Position-Dependent Deuteriation Effects on the Non-Radiative Decay of the ${ }^{3} \mathrm{MLCT}$ State of TrisBipyridine Ruthenium(II) and the Identification of the Critical Acceptor Mode." in revision, to be resubmitted to J. Phys. Chem.

8. G.D. Danzer, K. Bajdor and J.R. Kincaid. "Resonance Raman and Time-Resolved Resonance Raman Studies of ${ }^{3}$ ML.CT-state Population in the Series of Ruthenium(II) Complexes with Bipyridine and tetra-Methyl Bipyridine", J. Raman Spectrosc., in press. 

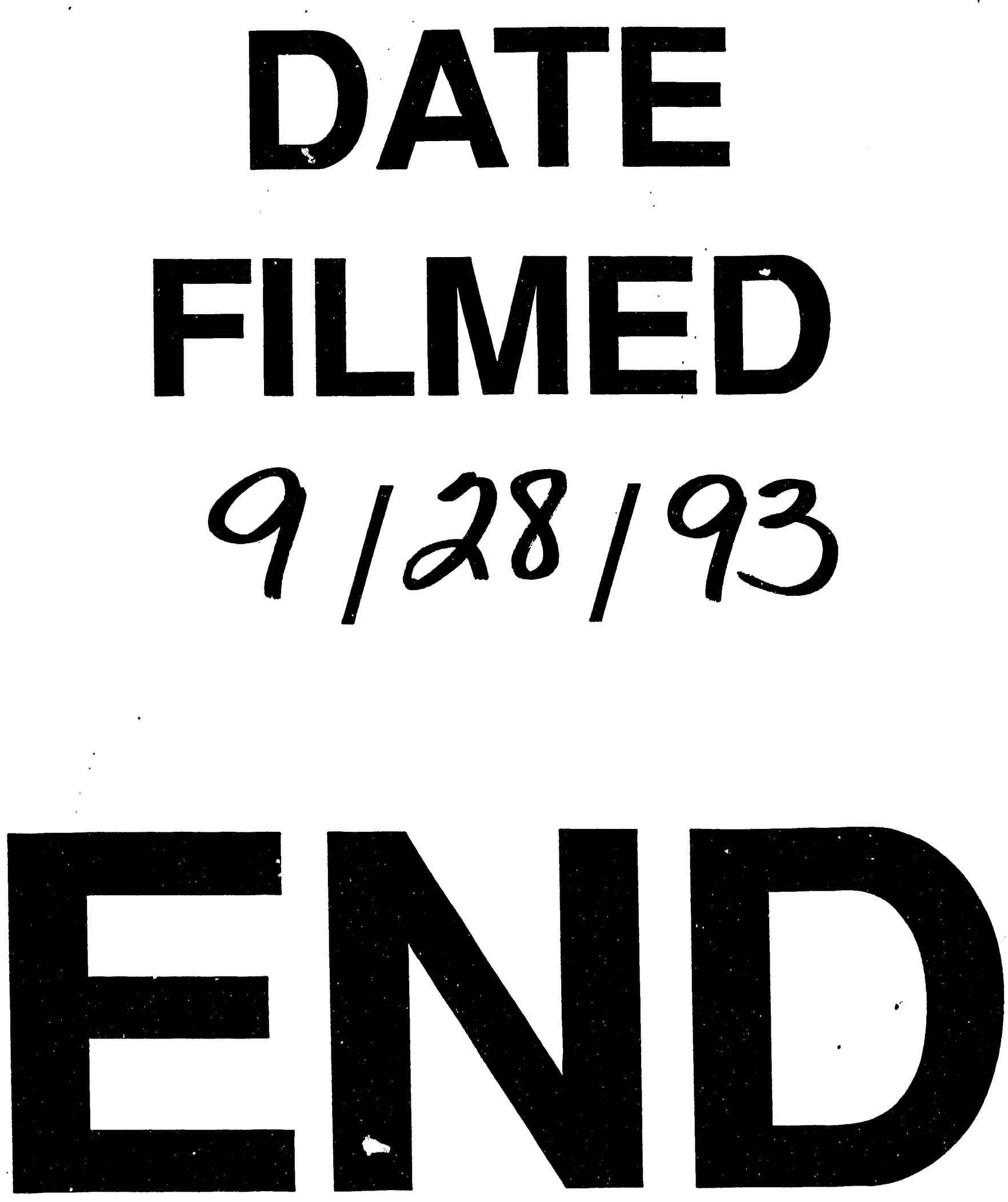
, $\quad$ 